\title{
Temporal set and cue selectivity in paired-associate learning accompanying changes of the stimulus component duration
}

\section{SUCHOON S. MO and KATHLEEN WARD University of Detroit, Detroit, Mich. 48221}

Cue selectivity of trigrams in PA learning is lessened by shortening of the duration of each stimulus component during the test, but no such lessening accompained lengthening of the duration of each stimulus component.

When each stimulus in PA learning consists of a horizontally displayed trigram, $S_{1} S_{2} S_{3}$, in sequence from left to right, so that $S_{1}$ tends to be more of a functional stimulus than the others, the question arises as to whether or not this "left-hand superiority" originates from a temporal ordering of the spatial sequence of the stimulus components and constitutes a case of primacy effect. This question calls for conceptual clarification of the temporal aspects of cue selectivity. Studies by Lovelace et al (Lovelace \& Blass, 1968; Lovelace \& Savage, 1972) imply that such left-hand superiority cannot be, in a strict sense, regarded as a case of primacy effect, because once $S_{1}$ is selected early in learning, $S_{2}$ and $S_{3}$ are mostly ignored. This implication is based on the evidence that left-hand superiority remains relatively invariant under various experimental conditions, specially the level of learning; it does not originate from an explicit manipulation of the stimulus duration itself. However, when dealing with the question of temporality in cue selectivity, the conceptual necessity of relating "which" not only to "when," but also to "how long" cannot be ignored. For instance, if $S_{1}$ is selected as a cue, it is as pertinent to ask when it is selected (primacy effect) as to ask for how long it remains selected. That is, the question of temporality in this case deals not only with period, but also with duration of cue selection. A simple and direct approach for this question would be to investigate the effect of changes of stimulus duration on cue selectivity.

Suppose that stimuli are presented for a fixed duration during learning, and that the duration is shortened when testing for the cue effectiveness of each stimulus component. If association of $S_{1}$ with the initial period of the stimulus duration is the critical factor, then shortening of the duration would not alter the cue effectiveness of $S_{1}$ as much as that of $S_{2}$ or $S_{3}$, for the shortened duration still contains the intital period of the original duration. Therefore, the left-hand superiority, if it is a case of primacy effect, would be left unaltered. On the other hand, if $S_{1}$ remains as a cue throughout the stimulus duration, shortening of the duration should decrease its cue effectiveness. On the other hand, the assumption that $S_{2}$ and $S_{3}$ remain selected for shorter durations than $S_{1}$ leads to the prediction that shortening of the duration should decrease their cue effectiveness to a less extent than $S_{1}$. Consequently, the left-hand superiority should be diminished. These predictions were tested.

\section{EXPERIMENT I \\ Method}

Twenty undergraduate volunteers were assigned to two groups of $10 \mathrm{Ss}$ each. Each stimulus consisted of three arbitrarily chosen consonants printed horizontally, doule-spaced, in capital letters. There were five stimuli. Responses were five single digits from 1 to 9 .

Each $S$ was instructed verbally at the beginning of the experimental session as to the task of associating each stimulus with its corresponding response, and was told that the subsequent test would be conducted using stimulus components. Each learning trial consisted of presenting the stimulus for $.5 \mathrm{sec}$ by means of a Lafayette U-1 tachistoscope, immediately followed by the verbal presentation of the response. For all the Ss, each S-R pair was presented three times, so that there were, altogether, 15 learning trials. The test was given following a brief rest. For one group, each stimulus component was tested for correct response twice, once using the component duration of $.5 \mathrm{sec}$ and once using $.1 \mathrm{sec}$, so that there were, altogether, 30 test trials presented randomly. For the other group, the test stimulus component durations were .5 and $.9 \mathrm{sec}$. The intertrial interval, that is, the rate of presentation, ranged between about 5 to $8 \mathrm{sec}$ as determined by the total running time.

\section{Results}

Figure 1 shows the proportions of correct response with respect to the stimulus components $S_{1}, S_{2}$, and $S_{3}$ in the order of letter positions from left to right. As for the group which was tested with the durations of .5 and $.9 \mathrm{sec}$, it is observed that the left-hand superiority, as manifested by enhanced cue selectivity toward left $(F=4.41, \mathrm{df}=2 / 45, \mathrm{p}<.025)$, is little affected by the lengthening of the duration. On the other hand, shortening the duration from .5 to $.1 \mathrm{sec}$ seems to affect the left-hand superiority to the extent that overall cue selectivity is lessened. But the results of the analysis of variance conducted within this group failed to show a significant interaction between the effects of letter position and the duration of the test stimulus components. It is possible that presentation of the $.5-\mathrm{sec}$ duration during the test exerted a contextual effect which lessened the generalization decrement accompanying the shortening of the duration. If so, elimination of this $.5-\mathrm{sec}$ duration during the test should disrupt the left-hand superiority to a greater extent.

\section{EXPERIMENT II Method}

This procedure differs from that of the previous experiment in two aspects. First, the learning trials were given until each $S$ made errorless responses to all the five stimuli themselves. This was accomplished by presenting each S-R pair three times in random order, followed by the test using stimuli. In case of error, learning trials were resumed and a "study-test" procedure was applied until no error response was made. Second, the .5-sec duration was eliminated from the test, so that each stimulus component was tested for a correct response using .1- and .9-sec durations. Otherwise, the general procedure was the same as in the previous experiment. Ten undergraduate volunteers served as Ss. 


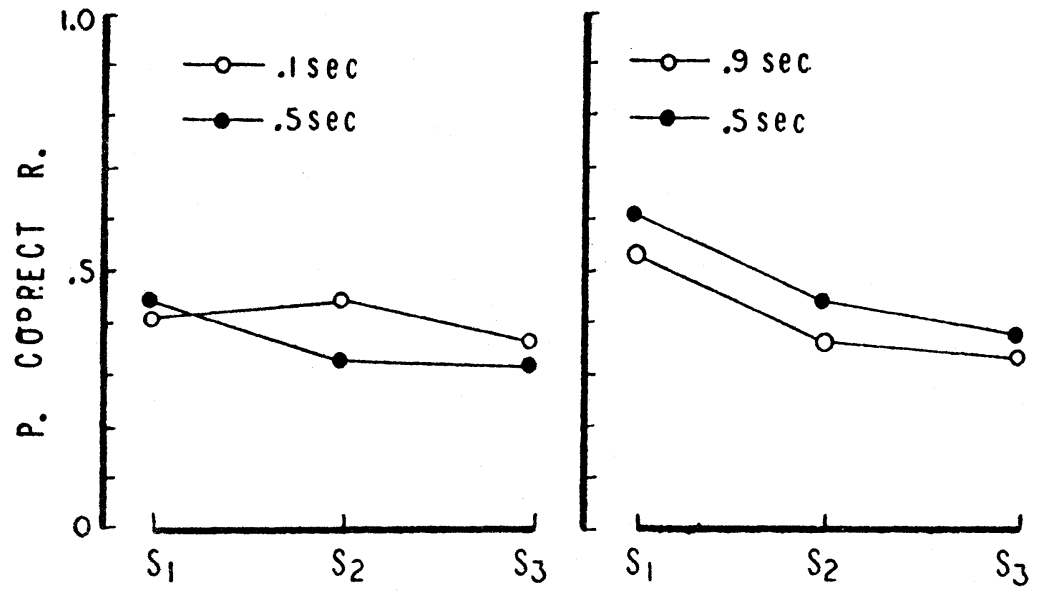

Fig. 1. Proportions of correct response with respect to the letter positions, $S_{1}, S_{2}$,

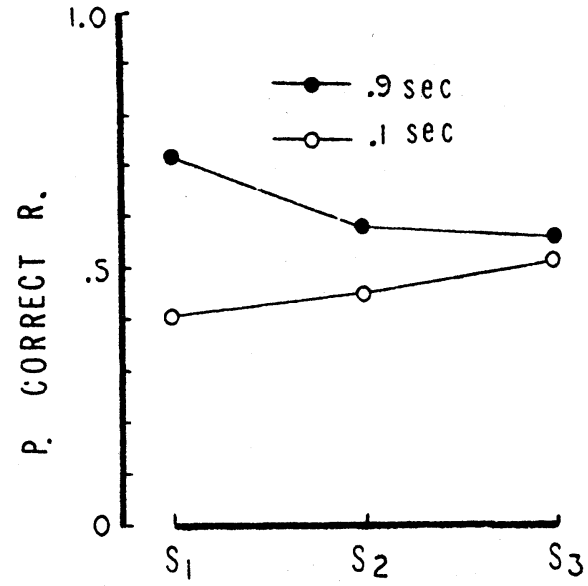

Fig. 2. Proportions of correct response with respect to the letter positions, $S_{1}, S_{2}$, and $S_{3}$. and $S_{\mathbf{3}}$.

\section{Results}

Figure 2 shows the proportions of the correct response with respect to the letter positions. It is noted that the proportions of correct response associated with the $.1-\mathrm{sec}$ are generally less than those associated with the .9-sec test stimulus component duration ( $F=5.83$, $\mathrm{df}=1 / 45, \mathrm{p}<.025)$, and that the effect of the direction of duration changes is the greatest at the first letter position, becoming more or less indistinguishable at the last position $(F=3.71, \mathrm{df}=2 / 45, \mathrm{p}<.05)$. Furthermore, when the duration is shortened, the cue effectiveness of stimulus components does not show a decreasing trend as a function of the letter positions; instead, such a trend seems to become an increasing one. This observation is in agreement with the original prediction that the shortening of duration would decrease the cue effectiveness of $S_{1}$ more than of $S_{2}$ or $\mathrm{S}_{3}$.

\section{DISCUSSION}

In general, the demonstrated phenomena do not render the concept of primacy effect, or any concept dealing only with the temporal period of cue selection, acceptable. Instead, the relevant temporal aspect seems to be that of the duration for which each stimulus component remains functioning as a cue. For the sake of clarification, the following specific explanation is made. When a stimulus is presented for a fixed duration during learning, $S_{1}$ is selected at the initial period and remains selected for the entire stimulus duration, while $S_{2}$ and $S_{3}$ are selected at subsequent periods and remain selected for the rest of their respective stimulus durations. Or it is irrelevant, even though scanning is assumed to be parallel, so that $S_{1}, S_{2}$, and $S_{3}$ are all selected at the same period; it is necessary only that $S_{1}$ remain selected for a longer duration than $S_{2}$ or $S_{3}$. Repeated presentation of the stimulus should result in the association of each stimulus component with a psychological process, possibly a preparatory set, specific to the duration for which each stimulus component remains selected. This duration-specific process may be called a temporal set. When each stimulus component is tested with the shortened duration, this temporal set associated with $S_{1}$ should be disrupted the most, resulting in elimination of the left-hand superiority. On the other hand, lengthening of the duration should not disrupt such a set.

Although this explanation in terms of a temporal set is tentative, performance decrement accompanying shortening of the stimulus duration is a generalized phenomenon which has been demonstrated in such a wide range of experiments as Pavlovian conditioning (Kimmel \& Greene, 1964; Kimmel, 1966), reaction time experiments (Karlin, 1959; Drazin, 1961; Zahn \& Rosenthal, 1966), and psychophysical recognition (Mo \& Jesky, 1971). That a similar phenomenon does occur in PA learning implies that the temporal process underlying the cue selectivity originating from a spatial sequence is not unique to PA learning itself. In this sense, also, temporal set is a reasonable terminology to use.

This study does not contradict any explanation based on an assumed serial scanning; it merely points to the insufficiency of such an explanation. Since scanning is defined in terms of periods of cue selection, whether scanning is serial or parallel does not bear upon the temporal aspect of cue selectivity. The fact that the response items were not of the same sense modality as stimuli renders an explanation of cue selectivity in terms of temporal period ambiguous.

The possibility that $S_{2}$ and $S_{3}$ are not selected, but merely ignored, is not counter to the concept of temporal set; it merely points to the argument that $S_{2}$ and $S_{3}$ are selected for a negligible duration. It is feasible to conceptualize the relevent temporal aspect of cue selectivity in terms of "how long" but not in terms of "when."

\section{REFERENCES}

Drazin, D. H. Effects of foreperiod, foreperiod variability, and probability of stimulus occurrence on simple reaction time. Journal of Experimental Psychology, 1961, 62, 43-50.

Karlin, L. Reaction time as a function of foreperiod duration and variability. Journal of Experimental Psychology, 1959, $58,185-191$.

Kimmel H. D. Inhibition of unconditioned response in classical conditioning. Psychological Review, 1966, 73, 232-240.

Kimmel, H. D., \& Greene, W. A. Disinhibition in GSR conditioning as a function of the number of CS-UCS trials and temporal location of the novel stimulus. Journal of Experimantal Psychology, 1964, 68, 567-572.

Lovelace, E. A., \& Blass, E. M. Utilization of stimulus elements in paired-associate learning. Journal of Experimental Psychology, 1968, 76, 596-600.

Lovelace, E. A., \& Savage, J. D. Stimulus encoding selectivity and degree of $P$-A learning. Paper presented at the annual meeting of the Psychonomic Society, 1972, St. Louis.

Mo, S. S., \& Jesky, L. Temporal set associated with brief stimulus duration in a psychophysical recognition of length. Psychonomic Science, 1971, 22, 337-338.

Zahn, $P$. T \& Rosenthal, D. Simple reaction time as a function of the relative frequency of the preparatory interval. Journal of Experimental Psychology, 1966, 72, 15-19.

(Received for publication April 5, 1973.) 